\title{
Vaginal metastasis presenting as postmenopausal bleeding
}

Qiu Ju Ng${ }^{1}$, MBBs, Rama Padma Namuduri ${ }^{2}$, MBBS, MRCoG, Kwai Lam $\underline{\text { Yam}}^{2}$, MRCoG, FAMs, Soo Kim $\underline{\operatorname{Lim}-\operatorname{Tan}^{3}}$, MBBS, FRCPath

\begin{abstract}
Vaginal cancer is rare worldwide and represents $2 \%$ of all gynaecological cancers in Singapore. Primary vaginal malignancies are rare and vaginal metastases constitute the majority of vaginal malignancies. Most of these metastases arise from the cervix, endometrium or ovary, although they can also metastasise from distant sites such as the colon, breast and pancreas. We report a rare case of vaginal metastasis in a patient with previous gastric and rectal adenocarcinomas. An 89-year-old woman with a history of gastric and rectal malignancy presented with postmenopausal bleeding. A 2-cm vaginal tumour at the introitus was discovered upon examination. This case demonstrates the importance of performing a gynaecological examination during follow-up for patients with a history of malignancy. The prognosis for vaginal metastasis is poor, as it is often associated with disseminated disease. Depending on the extent of the lesions, radiotherapy or surgery can be considered.
\end{abstract}

Keywords: colon carcinoma, postmenopausal bleed, recurrence, vaginal metastasis

\section{INTRODUCTION}

Vaginal cancer is rare worldwide. In Singapore, it represents $2 \%$ of all gynaecological cancers. From 1998-2002, the incidence of vaginal cancer in Singapore was 0.5 per 100,000 women per year. ${ }^{(1)}$ Vaginal cancer is diagnosed primarily in women aged $\geq 65$ years, with a median age at diagnosis of 69 years. The most common site of occurrence is at the lower-third of the vagina. About $90 \%$ of vaginal cancers are squamous cell carcinomas, followed by clear cell adenocarcinomas and melanomas. However, primary vaginal malignancies are rare and vaginal metastases constitute the majority of vaginal malignancies. Most of these metastases arise from the cervix, endometrium or ovary, although there have been case reports of metastasis from distant sites, such as the colon, kidney, breast and even the pancreas. ${ }^{(2-12)}$ We report a rare case of vaginal metastasis in a patient with previous gastric and rectal adenocarcinomas.

\section{CASE REPORT}

An 89-year-old woman presented with postmenopausal bleeding, which started in March 2013 when she complained of occasional per vaginal spotting. The spotting worsened to heavy bleeding requiring a change of two sanitary pads per day. This was associated with the onset of nocturia about 7-8 times per night, incomplete voiding and mild stress incontinence, although there was no associated dysuria, haematuria or fever. The patient had previously undergone a subtotal gastrectomy for poorly differentiated gastric adenocarcinoma in 1999, as well as, an abdominoperineal resection and end colostomy for a tubulovillous rectal malignancy that had invaded the upper anal canal and mesorectal soft tissues in 2011.

A physical examination revealed a $2-\mathrm{cm}$ vaginal tumour at the introitus; it was firm in consistency with contact bleeding

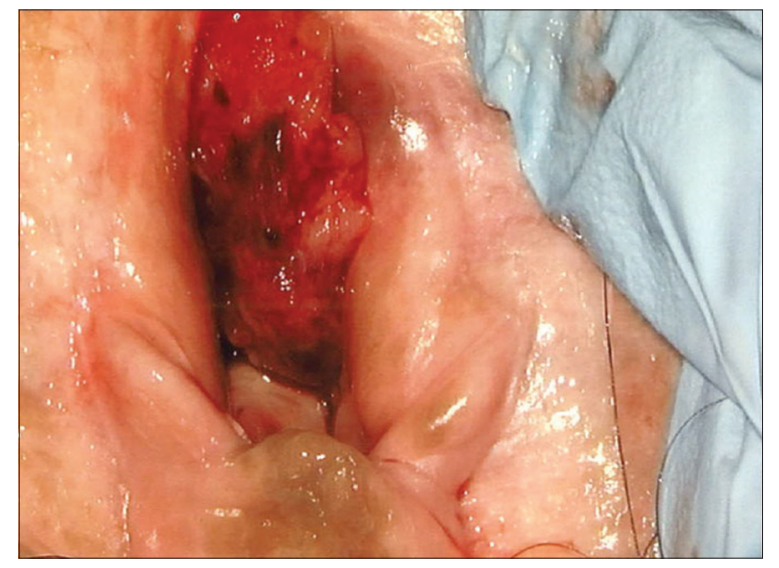

Fig. 1 Photograph shows a vaginal tumour at the introitus.

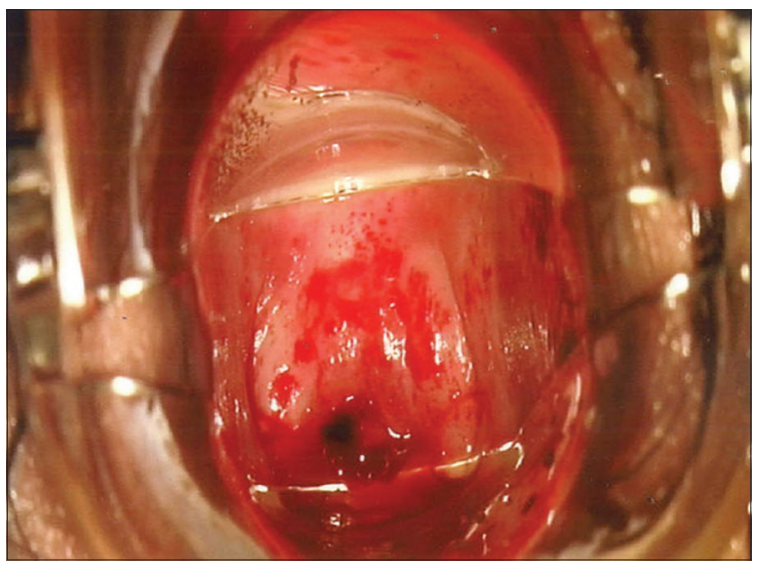

Fig. 2 Photograph shows an atrophic cervix.

(Fig. 1). The cervix, vulva and the rest of the vagina were atrophic (Fig. 2), and an excisional biopsy of the cervix was taken to rule out cervical causes of postmenopausal bleeding. An endocervical polyp, measuring $0.5 \mathrm{~cm}$ in diameter, was also removed. Attempts

${ }^{1}$ Department of Obstetrics and Gynaecology, 2Department of Gynaecological Oncology, ${ }^{3}$ Department of Women's Pathology and Cytology, KK Women's and Children's Hospital, Singapore Correspondence: Dr Ng Qiu Ju, Medical Officer, Department of Obstetrics and Gynaecology, KK Women's and Children's Hospital, 100 Bukit Timah Road, Singapore 229899. qiuju89@gmail.com 
to obtain endometrial sampling were unsuccessful and no inguinal lymphadenopathy was noted.

Histological examination of the vaginal tumour showed an intestinal-type, moderately differentiated mucinous adenocarcinoma (Figs. 3 \& 4). This was different from previous histological results in which the histology of the gastric cancer (in 1999) was poorly differentiated adenocarcinoma and the histology of the rectal tumour (2009) was a tubulovillous tumour with high-grade dysplasia. Immunohistochemistry was positive for cytokeratin 20 (CK20) and p16, but negative for CK7. Hence, this tumour was most likely a relapse of the rectal carcinoma. Pap smear and cervical biopsy findings were normal, and the endocervical polyp was benign.

Further investigations in the form of contrast tomography (CT) of the abdomen and pelvis demonstrated an ill-defined soft tissue density in the lower-third of the vagina (Fig. 5). Magnetic resonance (MR) imaging of the pelvis revealed a lobulated mass of approximately $2.6 \mathrm{~cm} \times 1.4 \mathrm{~cm}$, involving the lower-third of the vagina within the region of the introitus. There was involvement of the posterior and lateral vaginal walls with thickening of the anterior vaginal wall. The mass was closely related to the urethra, with periurethral enhancement noted, thus raising suspicion of involvement (Fig. 6). Otherwise, there were no signs of recurrence at the primary sites.

Due to a lack of consensus regarding the management of vaginal metastases from colorectal cancer, management is often tailored according to the patient's clinical condition. Chemotherapy is offered to patients with multiple metastatic sites, while surgical resection is the option for local control. ${ }^{(13)}$ Hence, chemotherapy was not offered to this patient, who had a single metastatic site. In view of the patient's advanced age and her significant history of two previous cerebrovascular accidents, she was a poor surgical candidate and the decision was made for conservative management in the form of radiotherapy. The patient completed ten sessions of radiotherapy, which caused a reduction in tumour size, and no further episodes of postmenopausal bleeding were encountered. Subsequently, the patient developed fresh bleeding from the colostomy and was found to have a hepatic flexure lesion, likely due to recurrence of the rectal cancer. The family decided against further workup or palliative radiotherapy in view of the patient's age. She was recommended for best supportive care and placed under home hospice follow-up.

\section{DISCUSSION}

Secondary vaginal cancers are more common than primary vaginal cancers and constitute $80 \%$ of vaginal malignancies. ${ }^{(14}$ Most of them are due to local extension from gynaecological malignancies such as cervical or vulvar cancer. Other reported sites of origin include the colon, rectum, breasts and kidneys. The majority of vaginal metastatic adenocarcinomas in the upper-third and anterior wall arise from the upper genital tract, while the majority of those in the lower-third and posterior wall arise from the gastrointestinal tract. ${ }^{(15)}$ The origin of the vaginal metastasis can be established based on immunohistochemistry.

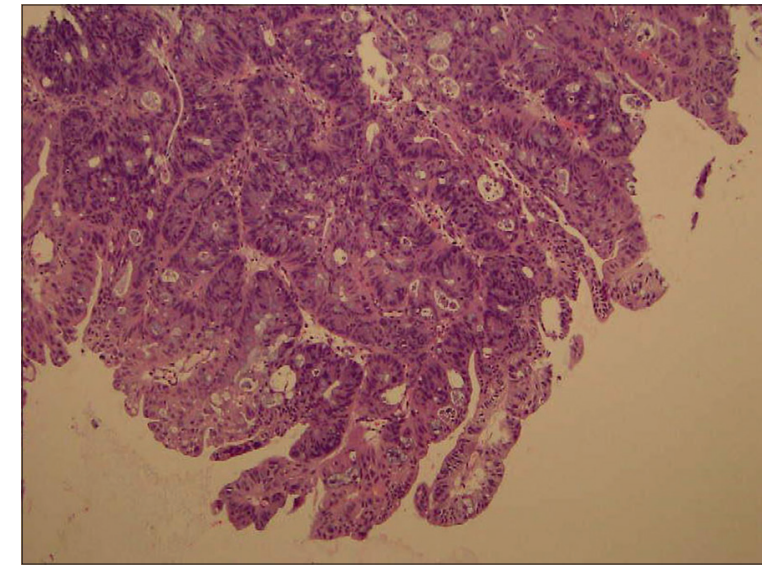

Fig. 3 Photomicrograph shows invasive glands with goblet cells (Haematoxylin \& eosin, $\times 100$ ).

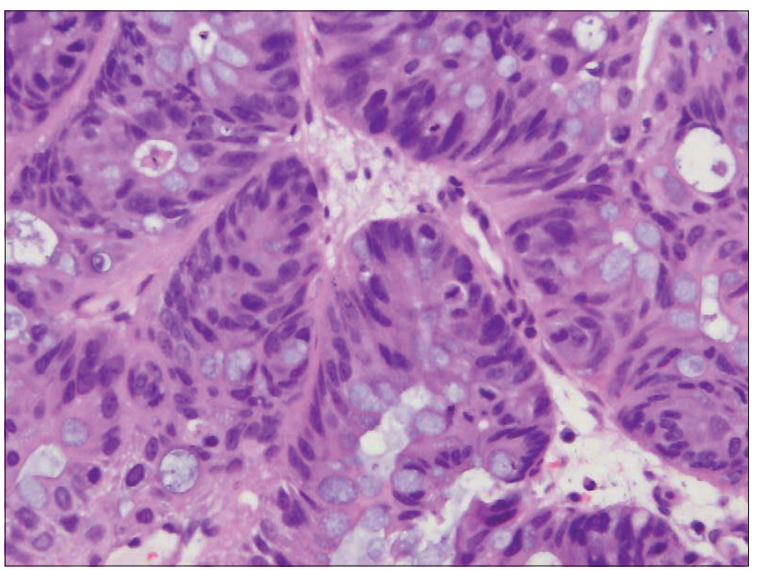

Fig. 4 Photomicrograph shows closely packed cells with scattered goblet cells (Haematoxylin \& eosin, $\times 400$ ).

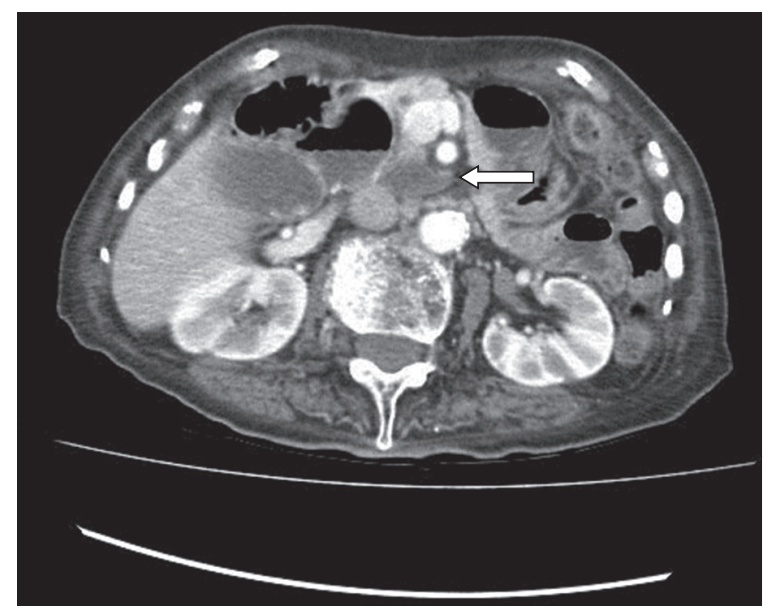

Fig. 5 CT image of the abdomen and pelvis shows a soft tissue density involving the lower-third of the vagina.

CK7 is present in tissues such as the lungs, breasts and vagina but is usually absent in the colon, whereas CK20 is almost exclusively present in the gastrointestinal tract. In our case, the vaginal metastasis is likely to be a recurrent colorectal carcinoma rather than a gastric carcinoma.

Vaginal metastases specifically from colorectal cancer are rare and carry a dismal prognosis, as they are associated with disseminated disease. ${ }^{(6-9)}$ The first case of vaginal metastasis from 

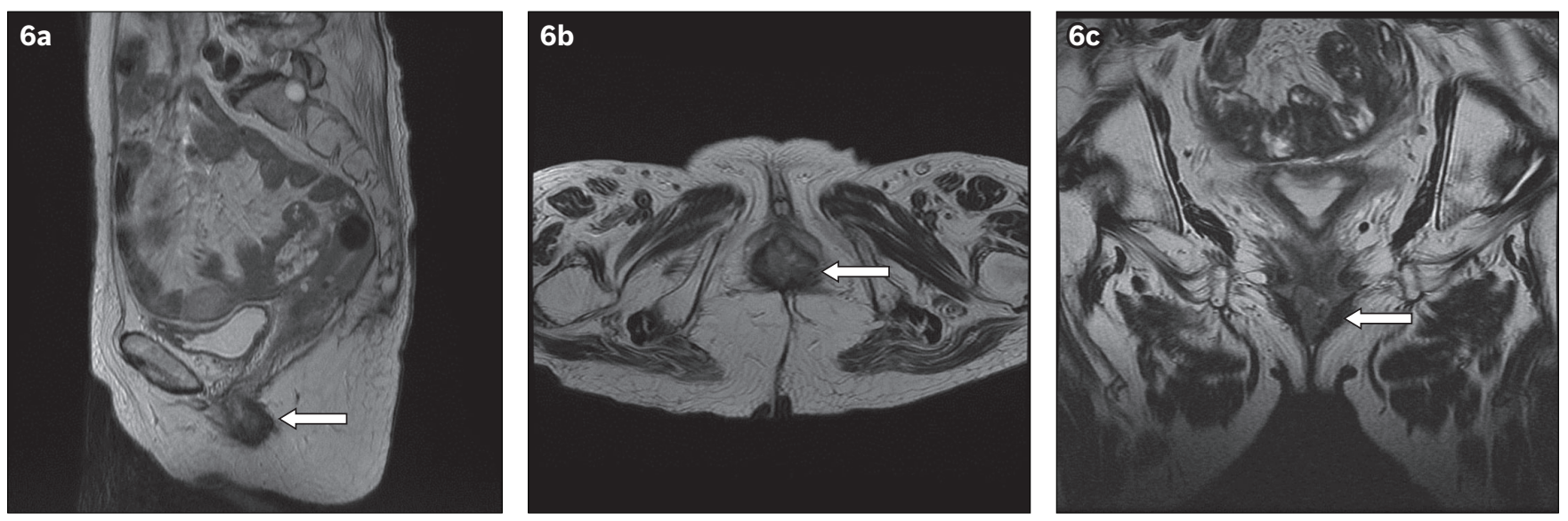

Fig. 6 MR images of the pelvis in (a) sagittal; (b) axial; and (c) coronal views show an approximately $2.6 \mathrm{~cm} \times 1.4 \mathrm{~cm}$ lobulated mass (arrows) involving the lower-third of the vagina at the region of the introitus. There is involvement of the posterior and lateral vaginal walls with thickening of the anterior vaginal wall.

colorectal cancer was reported by Whitelaw et $\mathrm{al}^{(10)}$ in 1956. Subsequently, Raider ${ }^{(6)}$ and Brand et $\mathrm{al}^{\left({ }^{(8)}\right.}$ also published case studies of vaginal metastases from primary colorectal cancer and anal cancer.

Various routes for the spread of malignancy to the vagina have been postulated, the most common being direct extension. Other routes include retrograde spread via the lymphatic channels, haematogenous spread via the venous plexus and transcoelomic spread. The lymph node drainage pattern of the vagina is such that lesions of the upper- and middle-thirds drain into the pelvic obturator nodes, internal and external iliac nodes and para-aortic nodes, while lesions of the lower-third drain into the inguinal nodes. Posterior wall lymphatics communicate with rectal lymphatics and drain into the inferior gluteal, sacral and rectal nodes. ${ }^{(16)}$ Hence, for our case, it is most likely a relapse of the rectal carcinoma via contiguous spread, as the rectal malignancy had invaded the mesorectal tissues and the lymph nodes were not involved.

Due to the limited number of reported cases of vaginal metastases from colorectal cancer, there is currently no guideline or consensus regarding its management options, which encompass surgical resection and adjuvant therapy in the form of radiotherapy and/or chemotherapy. Adjuvant therapy can potentially improve survival outcomes but its role requires further study. ${ }^{(13)}$ Currently, the therapeutic approach is via surgical resection for single vaginal lesion and radiotherapy, in the form of combined external beam therapy and interstitial brachytherapy. ${ }^{(7-9,11)}$ Chemotherapy is often used as an adjunct in patients with multiple metastatic sites. ${ }^{(13)}$

Our case demonstrated the importance of performing a thorough gynaecological examination during follow-up for patients with a history of malignancies, gynaecological or not, so as to detect recurrences early. The prognosis for vaginal metastasis is poor, as it is often associated with disseminated disease. Depending on the extent of the lesions, radiotherapy or surgery can be considered. In addition, it is important to rule out metachronous lesions when vaginal metastasis is present, as was evident in this case where the patient was subsequently found to have a hepatic flexure lesion.

\section{REFERENCES}

1. ICO HPV Information Centre. (Singapore) Human Papillomavirus and Related Cancers, Fact Sheet 2014. ICO Information Centre on HPV and Cancer; 2014 Dec.

2. Benbrahim Z, Chouaib A, Mazeron R, et al. Gynecologic bleeding revealing vaginal metastasis of renal cell carcinoma. Pan Afr Med J 2013; 14:62.

3. Weitzner S, Dressner SA. Vaginal metastasis from adenocarcinoma of pancreas. Am Surg 1974; 40:256-8.

4. Baba $\mathrm{H}$, Kuwabara $\mathrm{H}$, Wakabayashi $\mathrm{M}$, et al. [A case of vaginal metastasis of rectal cancer post -operation]. Gan To Kagaku Ryoho 2012; 39:2255-7. Japanese.

5. Funada T, Fujita S. A case of vaginal metastasis from a rectal cancer. Jpn J Clin Oncol 2010; 40:482.

6. Raider L. Remote vaginal metastases from carcinoma of the colon. Am J Roentgenol Radium Ther Nucl Med 1966; 97:944-50.

7. Chagpar A, Kanthan SC. Vaginal metastasis of colon cancer. Am Surg 2001; 67:171-2.

8. Brand A, Scurry J, Planner R, Leung S. Primary and recurrent colorectal cancer masquerading as gynaecological malignancy. Aust N Z J Obstet Gynaecol 1996; 36:165-7.

9. Costa SRP, Antunes RCP, Abraão AT, et al. Single vaginal metastasis from cancer of the right colon: case report. Einstein 2009;7(2 Pt 1):219-21.

10. Whitelaw GP, Leard SE, Parsons L, Sherwin RP. Carcinoma of the large bowel with metastasis to the genitalia; report of two cases. AMA Arch Surg 1956; 73:171-8

11. Marchal F, Leroux A, Hoffstetter S, Granger P. Vaginal metastasis revealing colon adenocarcinoma. Int J Colorectal Dis 2006; 21:861-2.

12. Yagci G, Cetiner S, Dede M, Gunhan O. True vaginal metastasis of rectal cancer. Indian J Surg 2005; 67:270-2.

13. Ng HJ, Aly EH. Vaginal metastases from colorectal cancer. Int J Surg 2013; 11:1048-55.

14. Merino MJ. Vaginal cancer: the role of infectious and environmental factors. Am J Obstet Gynecol 1991; 165(4 Pt 2):1255-63.

15. Outwater EK. CT and MRI of neoplasms metastatic to the genital tract. In: Anderson JC, eds. Gynaecologic Imaging. London, England: Churchill Livingstone, 1999: 519-34.

16. Parikh JH, Barton DP, Ind TE, Sohaib SA. MR imaging features of vaginal malignancies. Radiographics 2008; 28:49-63. 